\title{
A Comparative Study of Electromyographic Activity of Pectoralis Major and Anterior Deltoid Muscles During Intra-shoulder Rotation of Open and Closed Motion Chains in Normal Subjects: A Cross-sectional Observational Study
}

\author{
Amit Navani ${ }^{1}$, Hardik Trivedi ${ }^{2}$ \\ ${ }^{1}$ PT in Musculoskeletal and Sports Conditions, Assistance Professor, Shri K. K. Sheth Physiotherapy College, Rajkot, India \\ amitphysio44@gmail.com
}

${ }^{2}$ Incharge principal, Shree K. K. Sheth Physiotherapy College, Rajkot, India

\begin{abstract}
Background and objectives: One particularly vulnerable location for increased injury is the shoulder girdle. The shoulder girdle musculature provides the upper extremity with both mobility and stability. Due to the large amount of range of motion the shoulder is predisposed to overuse injuries. In the clinical setting and in the rehabilitation literature, functional activities and exercises are commonly categorized as having weight - bearing or non - weight - bearing characteristics. Another frequently used method of classifying movements and exercises is based on open or closed kinetic chain. The purpose of study was to compare the effects of open kinetic chain versus close kinetic chain exercises on muscle recruitment of the shoulder in healthy individuals. Method: 55 normal individuals with age ranging from 18 - 38 year old, who fulfilled the inclusion and exclusion criteria, were recruited by using random sampling method. EMG activity was recorded first in close kinetic chain exercise for pectoralis major and anterior deltoid muscles. Then in close kinetic chain exercise for pectoralis major and anterior deltoid muscles. Two trails were done and average of both trails was taken as consideration. Results: Data were analyzed by SPSS software 20.0; parametric Wilcoxen signed - rank test and Mann Whitney $U$ test were used. Result shows extremely significant difference between open kinetic chain and close kinetic chain exercise. $(p<0.05)$. Conclusion: The study concluded that there was extremely significant difference and greater surface electromyographic activity of the pectoralis major muscle as compared to anterior deltoid muscle in open kinetic chain and close kinetic chain exercise.
\end{abstract}

Keywords: EMG, open kinetic chain exercise, close kinetic chain exercise

\section{Introduction}

The upper - limb motions are very important for the human daily activities, such as eating, drinking, brushing teeth, combing hair and washing face. [1] The shoulder girdle connects the upper limb to the axial skeletal. The shoulder joint is formed by articulation of scapula and the head of humerus. Shoulder joint is the most freely mobile joint. It get excessive mobility at the cost of its own stability, since both are not feasible to the same degree. [2] Structurally it is weak joint because the glenoid cavity is too small and shallow to hold the head of humerus in place. [2] The maximum range of motion of human body lies in shoulder joints, it indicated that shoulders have over 1600 kind of position in three dimensions and the activity model of muscles of scapula are correspondingly complex. [3]

The pectoralis major has two distinct bellies, a smaller clavicular portion and a much larger sternal portion, each named for its proximal attachment. Effects of weakness must be hypothesized and include diminished strength in flexion and adduction of the shoulder. Effects of tightness also must be hypothesized and presumably include decreased ROM in abduction and extension of the shoulder. [4]
The deltoid is divided into three parts: anterior, middle, and posterior. The expansion of the acromion in humans increases the mechanical advantage of the deltoid as the distal migration of the deltoid tuberosity effectively increases the deltoid's contractile length. These changes improve the deltoid's ability to move the glenohumeral joint through its large available range of motion. [4]

Due to the large amount of range of motion (ROM) the shoulder is predisposed to overuse injuries. the muscles of the shoulder girdle complex are a frequent site of rehabilitation. [5]

The basis for the concept of kinetic chain originated from the field of engineering mechanics. Initial publications date back to Reuleaux in 1875 , who studied the coupling of various movements, he understood the combination of movements of various single components as rigid, overlapping segments that are interconnected via pin joints. These joints created a system, whereby a movement in one joint produced or affects movements at other joints in the kinetic link. Subsequent scientific discussions focused increasingly on the use of the terms 'kinetics' and 'kinematics'. These concepts, which were introduced during the 1950s and 1960s in the human biomechanics and 
kinesiology literature by Steindler and Brunnstrom, respectively. [6]

Open - chain exercises involve motions in which the distal segment (hand or foot) is free to move in space, without necessarily causing simultaneous motions at adjacent joints. Limb movement only occurs distal to the moving joint. Muscle activation occurs in the muscles that cross the moving joint. Open - chain exercise is typically performed in nonweight - bearing positions. [7] Closed - chain exercises involve motions in which the body moves on a distal segment that is fixed or stabilized on a support surface. Movement at one joint causes simultaneous motions at distal as well as proximal joints in a relatively predictable manner. Closed - chain exercises are primarily performed in weight bearing positions. [7]

Lee Herrington said altered dynamic control around the shoulder complex has been shown to be a significant factor in shoulder dysfunction. The balance of muscle force couples around the shoulder complex has been shown to be more important than muscle strength to establish normal joint function. [8]

Selective strengthening of the shoulder muscles has been emphasized as improving shoulder stability and preventing shoulder injuries in clinical and sports - related setting. [9] The purpose of study was to compare the effects of open kinetic chain versus close kinetic chain exercises on muscle recruitment of the shoulder in healthy individuals.

\section{Aim of the Study}

The aim of the study is to compare the electromyographic activities of pectoralis major and anterior deltoid muscles during shoulder internal rotation under open kinetic chain (OKC) and closed kinetic chain (CKC) exercise in normal healthy individuals.

\subsection{Objectives of the study}

1) To find the EMG activity of pectoralis major muscle in close kinetic chain exercise.

2) To find the EMG activity of anterior deltoid muscle in close kinetic chain exercise.

3) To find the EMG activity of pectoralis major muscle in open kinetic chain exercise.

4) To find the EMG activity of anterior deltoid muscle in open kinetic chain exercise

\subsection{Hypothesis}

\section{Null Hypothesis:}

There is no difference in electromyographic activity of pectoralis major and anterior deltoid muscles in open kinetic chain exercise and close kinetic chain exercise.

\section{Alternate hypothesis:}

There is a difference in electromyographic activity of pectoralis major and anterior deltoid muscles in open kinetic chain exercise and close kinetic chain exercise.

\section{Material and Methodology}

\section{Method of collection of data}

Study Design: A cross - sectional

Observational Study

Sampling Technique: Random sampling method

Sample Size: 55 subjects

Study Population: Normal healthy males and females

Source of Data: Subjects were selected from Rajkot city.

Study Duration: One - time study

\section{Criteria for selection}

\section{Inclusion criteria:}

- Normal healthy subjects

- Age group - 18 to 38 years

- Dominant side of the subject

- Gender - male and female are taken

\section{Exclusion criteria:}

- Any trauma or musculoskeletal disorders related to upper limb or neck region on dominant side

- Any neurological conditions related to upper limb or neck region

- Any surgery done on dominant side within last 6 months

- Any skin disorder

- Un co - operative person

After the approval of the study from the ethical committee, 55 subjects were taken, who fulfilled the inclusion and exclusion criteria taken for the study purpose. After that explanation about the purpose and procedure of this study, subjects who were willing to participate in this study were requested to sign a written consent form. The selection of subjects was done by simple Random Sampling procedure. A pre - participation evaluation form consisting of basic musculoskeletal assessment chart was filled. The data measured were recorded in the measurement form which included name, age, gender, occupation, address, dominance side, Patients were then explained about the test and procedure to be conducted.

\section{Result and Tables}

All the statistical analysis was done by Statistical Package for the Social Sciences (SPSS) statistical software version 20.0 for windows. Microsoft excel was used to generate graphs and tables. Mean and standard deviation were calculated as measure of central tendency and measure of dispersion respectively. Normality of data was checked by using Kolmogorov - Smirnov test which shows that data is of non - parametric type. Level of significance ( $p$ value) was set to 0.05 level. 
Table 1: Comparison between pectoralis major and anterior deltoid muscles in open kinetic chain exercise

\begin{tabular}{|l|l|l|l|l|l|l|l|l|}
\hline Position & Muscle & N & Mean & SD & Min. & Max. & Z Value & p \\
\hline \multirow{2}{*}{ Open Kinetic Chain Exercise } & Pectoralis Major & 55 & 306.29 & 151.69 & 131.50 & 1158.00 & \multirow{2}{*}{-4.881} & \multirow{2}{*}{0.000} \\
\cline { 2 - 8 } & Anterior Deltoid & 55 & 211.73 & 153.70 & 57.50 & 873.00 & \\
\hline
\end{tabular}

Table 2: Comparison between pectoralis major and anterior deltoid muscles in close kinetic chain exercise

\begin{tabular}{|l|l|l|l|l|l|l|l|l|}
\hline Position & Muscle & N & Mean & SD & Min. & Max. & Z Value & p \\
\hline \multirow{2}{*}{ Close Kinetic Chain Exercise } & Pectoralis Major & 55 & 375.67 & 176.74 & 170.50 & 1281.50 & -4.072 & 0.000 \\
\cline { 2 - 10 } & Anterior Deltoid & 55 & 288.31 & 185.52 & 61.50 & 906.00 & & \\
\hline
\end{tabular}

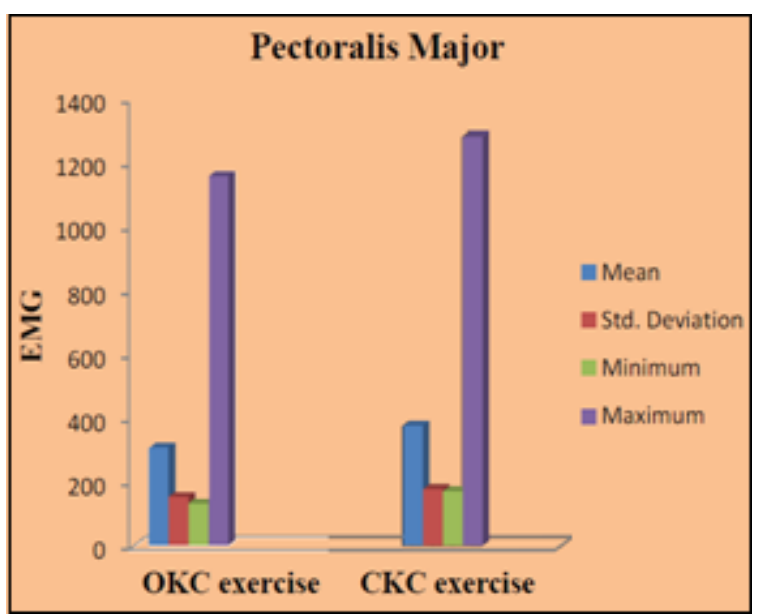

Graph: Shows comparison between open kinetic chain and close kinetic chain exercise in pectoralis major muscle.

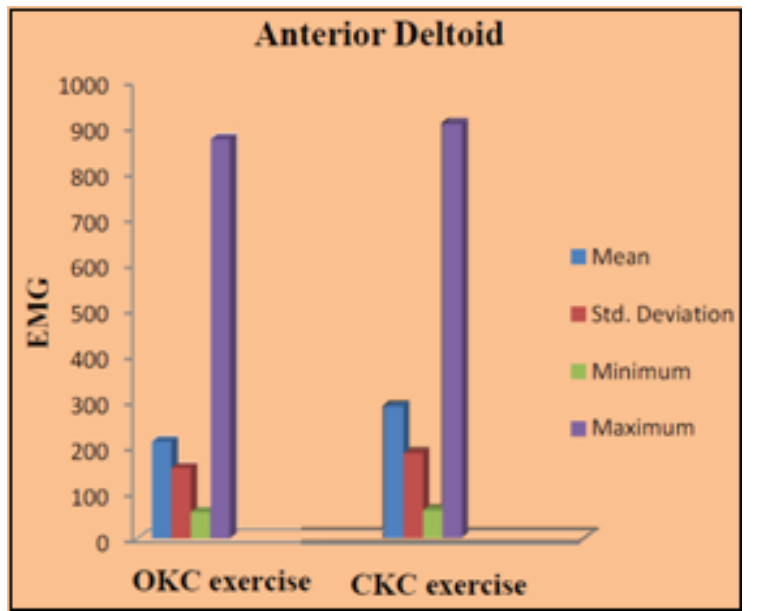

Graph: Shows comparison between open kinetic chain and close kinetic chain exercise in anterior deltoid muscle

\section{Discussion}

The results of the present study support the alternate hypothesis which states that there is highly significant difference between EMG activities of pectoralis major and anterior deltoid in open kinetic chain and close kinetic chain exercise. The present study found that close kinetic chain during shoulder internal rotation exercise is more effective at selectively strengthening the pectoralis major muscle than open kinetic chain in shoulder internal rotation exercise.

In open kinetic chain exercise, highly significant difference was found on comparing both muscles. This result suggest that while performing open kinetic chain exercise the pectoralis major muscle works more as compared to anterior deltoid muscle. As the shoulder works mostly in open kinetic chain position and in adduction position, indicate that pectoralis major muscle remains active more than deltoid muscles. Lee Herrington et al found muscle activation timing in rugby player and results indicate that timing of muscle activation is not properly balanced around the shoulder girdle during excessive load and stress. [8]

In close kinetic chain exercise also, highly significant difference was found on comparing both muscles. This result suggest that while performing close kinetic chain exercise the pectoralis major work more as compared to anterior deltoid muscle. Gregory J Lehman et al found that by replacing an exercise bench for a Swiss ball can increase muscle activity however, the effect is both task and muscle dependent. [10]

For pectoralis major muscle by comparing in open kinetic chain and closed kinetic chain the significant difference was found. The activity of pectoralis major muscle is more in close kinetic chain exercise as compared to open kinetic chain exercise. Livingston et al found closed chain exercises should, therefore, be utilized in shoulder and scapula rehabilitation for functional return to most athletic activities from all types of shoulder injuries. Early in rehabilitation, when the shoulder muscles are weakest, facilitation needs from proximal muscle activation are at their greatest. [11]

For anterior deltoid muscle by comparing in open and closed kinetic chain the significant difference was found. The activity of anterior deltoid muscle is found more in close kinetic chain exercise as compared to open kinetic chain exercise. Reinaldo Noguti et al found that the similar exercises classified by the extremity condition and the load direction applied to the upper limbs promote similar levels on the electromyographic activity in part of the assessed muscles. [12]

In this study, the athlete subjects were included which shows increase in activities of pectoralis major and anterior deltoid muscles than non - athlete person.

In present study, the pectoralis major muscle activity was significantly increased with less activity of the anterior deltoid during shoulder internal rotation exercise under the close kinetic chain and the open kinetic chain condition. The present findings suggest that the close kinetic chain exercise condition is more appropriate for maximizing the contribution of the pectoralis major and anterior deltoid in shoulder internal rotation exercises during shoulder rehabilitation in traumatic and pathological conditions. So, alternate hypothesis was accepted and null hypothesis was rejected. 


\section{Limitations of the Study}

- The study was conducted in normal healthy subjects.

- Only two muscles were used for the study.

- In regards to the use of surface EMG to measure muscle activity; the possibility of cross talk between muscles exists.

\section{Further Recommendation}

- Future research includes considering the other internal rotators muscles.

- Needle EMG can be used for other muscles.

- Future research includes the subjects with shoulder pathology conditions to find any abnormal activities of the muscles.

\section{Conclusion}

Depending upon this study it can be concluded that there was extremely significant difference and greater surface electromyographic activity of the pectoralis major muscle as compared to anterior deltoid muscle in open kinetic chain and close kinetic chain exercise.

Acknowledgement: I would like to thanks Dr. and Dr. for guidance. Also my parents $\&$ friends for their support

Conflict of Interest: There was no personal or institutional conflict of interest for this study.

Ethical Clearance: From K. K. Sheth Physiotherapy College, Rajkot.

\section{References}

[1] R. A. R. C. Gopura, Kazuo Kiguchi, Etsuo Horikawa. A study on human upper - limb muscles activities during daily upper - limb motions. International Journal of Bioelectromagnetism.2010; 12 (2): 54 - 61

[2] B D Chaurasia's. Human anatomy regional and applied dissection and clinical: Volume (1) Upper Limb and Thorax.5th edition. CBS publishers \& distributors. 2010

[3] Pei - Hung Wang, Fong - Gong Wu. Research of shoulder activities in range of motion. National Cheng Kung University. Sep - 2017.

[4] Carol A. Oatis. Kinesiology - The Mechanics and Pathomechanics of Human Movement.2nd Edition. Lippincott Williams \& Wilkins.2009.

[5] Johnstone, Kathleen and Wagner, Kathleen. Assessing selected shoulder muscle activity during performance of exercise on the cuff link exercise Unit. Masters These.1998; Paper 413.

[6] F. Mayer, A. Schlumberger, R. van Cingel, Y. Henrotin, W. Laube and D. Schmidtbleicher. Training and testing in open versus closed kinetic chain. Isokinetics and Exercise Science.2003; 11: 181 - 187.

[7] Carolyn Kisner and Lynn Allen Colby. Therapeutic exercise foundations and techniques.5th edition. New Delhi: Jaypee brother's medical publishers (p) Ltd; 2007.
[8] Lee Herrington, Ian Horsley. Electromyographic analysis of selected shoulder muscles during a rugby football tackle. Sports Medicine Arthroscopy Rehabilitation Therapy and Technology.2009; 1: 10

[9] Min - H. K, Jae S. O, Jun - H. J. Differences in muscle activities of the Infraspinatus and posterior deltoid during shoulder external rotation in open kinetic chain and closed kinetic chain exercises. J Phys Ther Sci.2014 Jun; 26 (6): 895-897.

[10] Gregory J Lehman, Brandon MacMillan, Ian MacIntyre Shoulder muscle EMG activity during push up variations on and off a Swiss ball. BioMed Central Ltd.2006 June.

[11] Kibler WB, Livingston B. Closed chain rehabilitation for upper and lower extremities. J Am Acad Orthop Surg.2001 Nov; 9 (6): 412 - 421.

[12] Anamaria Siriani de Oliveira, Carina Maria de Souza Freitas, Francisco Henriqu Monaretti, Francislei Ferreira, Fausto Bérzin Reinaldo Noguti. Electromyographic assessment of the shoulder girdle and arm muscles during exercises with axial and rotational loads. Rev Bras Med Esporte.2006 Jan; 12 (1). 